\title{
Analytical Solution of Nonlinear System of Fractional Differential Equations
}

\author{
Eman Ali Ahmed Ziada \\ Basic Science Department, Nile Higher Institute for Engineering \& Technology, Mansoura, Egypt \\ Email: eng_emanziada@yahoo.com
}

How to cite this paper: Ziada, E.A.A (2021) Analytical Solution of Nonlinear System of Fractional Differential Equations. Journal of Applied Mathematics and Physics, 9, 2544-2557.

https://doi.org/10.4236/jamp.2021.910164

Received: September 13, 2021

Accepted: October 25, 2021

Published: October 28, 2021

Copyright $\odot 2021$ by author(s) and Scientific Research Publishing Inc. This work is licensed under the Creative Commons Attribution International License (CC BY 4.0).

http://creativecommons.org/licenses/by/4.0/

(c) (i) Open Access

\begin{abstract}
In this paper, we apply the Adomian decomposition method (ADM) for solving nonlinear system of fractional differential equations (FDEs). The existence and uniqueness of the solution are proved. The convergence of the series solution and the error analysis are discussed. Some applications are solved such as fractional-order rabies model.
\end{abstract}

\section{Keywords}

Fractional Differential Equations, Adomian Decomposition Method, Existence, Uniqueness, Error Analysis, Rabies Model

\section{Introduction}

This paper is concerned with the analytical solution of a nonlinear system of fractional differential equations. Systems of fractional differential equations (FDEs) have many applications in engineering and science, including electrical networks, fluid flow, control theory, fractals theory, electromagnetic theory, viscoelasticity, potential theory, chemistry, biology, optical and neural network systems ([1]-[16]). We use Adomain decomposition method ([17]-[24]) for solving this type of equations. The existence and uniqueness of the solution are proved, the convergence of ADM series solution is discussed and the error analysis is given. This method has many advantages; it is efficiently working with different types of linear and nonlinear equations in deterministic or stochastic fields and gives an analytic solution for all these types of equations without linearization or discretization.

\section{Formulation of the Problem}

Consider the following nonlinear system of FDEs: 


$$
{ }_{0} \mathcal{D}_{t}^{\sigma_{n}} y_{i}(t)+\beta_{i}(t) f_{i}(\bar{y})=x_{i}(t),
$$

subject to the initial conditions,

$$
y_{i}^{(j-1)}(0)=c_{i j}, \quad i, j=1,2, \cdots, n .
$$

where,

$$
\begin{aligned}
\bar{y} & =\left\{y_{1}(t), y_{2}(t), \cdots, y_{n}(t)\right\}, \\
{ }_{0} \mathcal{D}_{t}^{\sigma_{n}} & \equiv{ }_{0} D_{t}^{\alpha_{n}}{ }_{0} D_{t}^{\alpha_{n-1}}{ }_{0} D_{t}^{\alpha_{n-2}} \cdots{ }_{0} D_{t}^{\alpha_{1}}, \\
\sigma_{n} & =\sum_{k=1}^{n} \alpha_{k}, n-1 \leq \alpha_{k} \leq n,
\end{aligned}
$$

where $x_{i}(t)$ is bounded $\forall t \in J=[0, T], T \in R^{+},\left|\beta_{i}(\tau)\right| \leq M_{i}$ $\forall 0 \leq \tau \leq t \leq T, M_{i}$ are finite constants and $f_{i}(\bar{y})$ satisfy Lipschitz condition with Lipschitz constants $L_{i}$ such as,

$$
\left|f_{i}(\bar{y})-f_{i}(\bar{z})\right| \leq L_{i}|\bar{y}-\bar{z}|
$$

and the fractional derivative in this system is of sequential Caputo sense which defined as

$$
{ }_{0} D_{t}^{\alpha} y(t)=\frac{1}{\Gamma(\alpha-n)} \int_{0}^{t} \frac{y^{(n)}(\tau) \mathrm{d} \tau}{(t-\tau)^{\alpha+1-n}}, \quad(n-1<\alpha<n),
$$

In the applications, the Caputo sense is preferred to be used because the initial conditions of $y_{i}(t)$ and its derivatives will be of integer orders and have a physical meaning.

Now performing subsequently the fractional integration of order $\alpha_{n}, \alpha_{n-1}, \cdots, \alpha_{1}$, this reduces the system (1)-(2) to the system of FIEs,

$$
\begin{aligned}
y_{i}(t)= & \sum_{j=1}^{n} \frac{c_{i j}}{\Gamma\left(\sigma_{j}\right)} t^{\sigma_{j-1}}+\frac{1}{\Gamma\left(\sigma_{n}\right)} \int_{0}^{t}(t-\tau)^{\sigma_{n-1}} x_{i}(\tau) \mathrm{d} \tau \\
& -\frac{1}{\Gamma\left(\sigma_{n}\right)} \int_{0}^{t} \beta_{i}(\tau)(t-\tau)^{\sigma_{n-1}} f_{i}(\bar{y}) \mathrm{d} \tau
\end{aligned}
$$

and has Adomian polynomials representation,

$$
f_{i}(\bar{y})=\sum_{k=0}^{\infty} A_{i k}\left(y_{i 0}, y_{i 1}, \cdots, y_{i n}\right)
$$

where,

$$
A_{i k}=\frac{1}{k !} \frac{\mathrm{d}^{k}}{\mathrm{~d} \lambda^{k}}\left[f_{i}\left(\sum_{j=0}^{\infty} \lambda^{j} y_{j}\right)\right]_{\lambda=0}
$$

Substitute from Equation (5) into Equation (3), we get

$$
\begin{aligned}
y_{i}(t)= & \sum_{j=1}^{n} \frac{c_{i j}}{\Gamma\left(\sigma_{j}\right)} t^{\sigma_{j-1}}+\frac{1}{\Gamma\left(\sigma_{n}\right)} \int_{0}^{t}(t-\tau)^{\sigma_{n-1}} x_{i}(\tau) \mathrm{d} \tau \\
& -\frac{1}{\Gamma\left(\sigma_{n}\right)} \int_{0}^{t} \beta_{i}(\tau)(t-\tau)^{\sigma_{n-1}} \sum_{k=0}^{\infty} A_{i k} \mathrm{~d} \tau .
\end{aligned}
$$

Let $y_{i}(t)=\sum_{k=0}^{\infty} y_{i k}(t)$ in (7) we get, 


$$
\begin{aligned}
& y_{i 0}(t)=\sum_{j=1}^{n} \frac{c_{i j}}{\Gamma\left(\sigma_{j}\right)} t^{\sigma_{j-1}}+\frac{1}{\Gamma\left(\sigma_{n}\right)} \int_{0}^{t}(t-\tau)^{\sigma_{n-1}} x_{i}(\tau) \mathrm{d} \tau, \\
& y_{i k}(t)=-\frac{1}{\Gamma\left(\sigma_{n}\right)} \int_{0}^{t} \beta_{i}(\tau)(t-\tau)^{\sigma_{n-1}} A_{i(k-1)} \mathrm{d} \tau, \quad k \geq 1 .
\end{aligned}
$$

Finally, the solution is,

$$
y_{i}(t)=\sum_{k=0}^{\infty} y_{i k}(t)
$$

\section{Analysis of Convergence}

\subsection{The Uniqueness of Solution}

In the previous section, we find the series solution (10) of the system (1)-(2) and here we want to prove the existence and uniqueness of this series solution.

Theorem 1. If $0<\alpha<1$ where $\alpha=\frac{L M T^{\sigma_{n}}}{\Gamma\left(\sigma_{n}+1\right)}$, then the series (10) is the solution of the system (1)-(2) and this solution is unique, where $L=\max \left\{L_{1}, L_{2}, \cdots, L_{n}\right\}, M=\max \left\{M_{1}, M_{2}, \cdots, M_{n}\right\}$.

Proof. For existence,

$$
\begin{aligned}
y_{i}(t)= & \sum_{k=0}^{\infty} y_{i k}(t)=y_{i 0}(t)+\sum_{k=1}^{\infty} y_{i k}(t) \\
= & y_{i 0}(t)-\frac{1}{\Gamma\left(\sigma_{n}\right)} \sum_{k=1}^{\infty} \int_{0}^{t} \beta_{i}(\tau)(t-\tau)^{\sigma_{n-1}} A_{i(k-1)} \mathrm{d} \tau \\
= & y_{i 0}(t)-\frac{1}{\Gamma\left(\sigma_{n}\right)} \int_{0}^{t} \beta_{i}(\tau)(t-\tau)^{\sigma_{n-1}} \sum_{k=1}^{\infty} A_{i(k-1)} \mathrm{d} \tau \\
= & y_{i 0}(t)-\frac{1}{\Gamma\left(\sigma_{n}\right)} \int_{0}^{t} \beta_{i}(\tau)(t-\tau)^{\sigma_{n-1}} \sum_{k=0}^{\infty} A_{i k} \mathrm{~d} \tau \\
= & \sum_{j=1}^{n} \frac{c_{i j}}{\Gamma\left(\sigma_{j}\right)} t^{\sigma_{j-1}}+\frac{1}{\Gamma\left(\sigma_{n}\right)} \int_{0}^{t}(t-\tau)^{\sigma_{n-1}} x_{i}(\tau) \mathrm{d} \tau \\
& -\frac{1}{\Gamma\left(\sigma_{n}\right)} \int_{0}^{t} \beta_{i}(\tau)(t-\tau)^{\sigma_{n-1}} f_{i}(\bar{y}) \mathrm{d} \tau
\end{aligned}
$$

then the Adomian's series solution satisfy Equation (4) which is the reduced system of FIEs to the system (1)-(2).

For uniqueness of the solution: Assume that $\bar{y}$ and $\bar{z}$ are two different solutions to the system (1)-(2) and hence,

$$
\begin{aligned}
|\bar{y}-\bar{z}| & =\left|\frac{1}{\Gamma\left(\sigma_{n}\right)} \int_{0}^{t} \beta_{i}(\tau)(t-\tau)^{\sigma_{n-1}}\left[f_{i}(\bar{y})-f_{i}(\bar{z})\right] \mathrm{d} \tau\right| \\
& \leq \frac{1}{\Gamma\left(\sigma_{n}\right)} \int_{0}^{t}(t-\tau)^{\sigma_{n-1}}\left|\beta_{i}(\tau)\right|\left|f_{i}(\bar{y})-f_{i}(\bar{z})\right| \mathrm{d} \tau \\
& \leq \frac{L_{i} M_{i}}{\Gamma\left(\sigma_{n}\right)}|\bar{y}-\bar{z}| \int_{0}^{t}(t-\tau)^{\sigma_{n-1}} \mathrm{~d} \tau
\end{aligned}
$$




$$
\begin{aligned}
& \leq \frac{L_{i} M_{i} T^{\sigma_{n}}}{\Gamma\left(\sigma_{n}+1\right)}|\bar{y}-\bar{z}| \\
& \leq \frac{L M T^{\sigma_{n}}}{\Gamma\left(\sigma_{n}+1\right)}|\bar{y}-\bar{z}|
\end{aligned}
$$

Let $\frac{L M T^{\sigma_{n}}}{\Gamma\left(\sigma_{n}+1\right)}=\alpha$ where, $0<\alpha<1$ then,

$$
\begin{aligned}
& |\bar{y}-\bar{z}| \leq \alpha|\bar{y}-\bar{z}| \\
& (1-\alpha)|\bar{y}-\bar{z}| \leq 0
\end{aligned}
$$

but, $(1-\alpha)|\bar{y}-\bar{z}| \geq 0$ and since, $(1-\alpha) \neq 0$ then, $|\bar{y}-\bar{z}|=0$ this implies that, $\bar{y}=\bar{z}$ and this completes the proof.

\subsection{Proof of Convergence}

Theorem 2. The series solution (10) of the system (1)-(2) using ADM converges if $\left|y_{i 1}\right|<\infty$ and $0<\alpha<1, \alpha=\frac{L M T^{\sigma_{n}}}{\Gamma\left(\sigma_{n}+1\right)}$, where $L=\max \left\{L_{1}, L_{2}, \cdots, L_{n}\right\}, M=\max \left\{M_{1}, M_{2}, \cdots, M_{n}\right\}$.

Proof. Define the Banach space $(C[J],\|\cdot\|)$, the space of all continuous functions on $J$ with the norm $\|y(t)\|=\max _{t \in J}|y(t)|$ and a sequence $\left\{S_{i n}\right\}$ such that, $S_{i n}=\sum_{k=0}^{n} y_{i k}(t)$. We have,

$$
f\left(S_{i n}\right)=\sum_{k=0}^{n} A_{i k}\left(y_{i 0}, y_{i 1}, \cdots, y_{i n}\right)
$$

Let, $S_{i n}$ and $S_{i m}$ be two arbitrary partial sums with $n \geq m$. Now, we are going to prove that $\left\{S_{i n}\right\}$ is a Cauchy sequence in this Banach space.

$$
\begin{aligned}
\left\|S_{i n}-S_{i m}\right\| & =\max _{t \in J}\left|S_{i n}-S_{i m}\right| \\
& =\max _{t \in J}\left|\sum_{k=m+1}^{n} y_{i k}(t)\right| \\
& =\max _{t \in J}\left|\sum_{k=m+1}^{n}-\frac{1}{\Gamma\left(\sigma_{n}\right)} \int_{0}^{t} \beta_{i}(\tau)(t-\tau)^{\sigma_{n-1}} A_{i(k-1)} \mathrm{d} \tau\right| \\
\left\|S_{i n}-S_{i m}\right\| & =\max _{t \in J}\left|\frac{1}{\Gamma\left(\sigma_{n}\right)} \int_{0}^{t} \beta_{i}(\tau)(t-\tau)^{\sigma_{n-1}} \sum_{k=m+1}^{n} A_{i(k-1)} \mathrm{d} \tau\right| \\
& =\max _{t \in J}\left|\frac{1}{\Gamma\left(\sigma_{n}\right)} \int_{0}^{t} \beta_{i}(\tau)(t-\tau)^{\sigma_{n-1}} \sum_{k=m}^{n-1} A_{i k} \mathrm{~d} \tau\right| \\
& =\max _{t \in J}\left|\frac{1}{\Gamma\left(\sigma_{n}\right)} \int_{0}^{t} \beta_{i}(\tau)(t-\tau)^{\sigma_{n-1}}\left[f\left(S_{i(n-1)}\right)-f\left(S_{i(m-1)}\right)\right] \mathrm{d} \tau\right| \\
& \leq \frac{1}{\Gamma\left(\sigma_{n}\right)} \max _{t \in J} \int_{0}^{t}(t-\tau)^{\sigma_{n-1}}\left|\beta_{i}(\tau)\right|\left|f\left(S_{i(n-1)}\right)-f\left(S_{i(m-1)}\right)\right| \mathrm{d} \tau \\
& \leq \frac{L_{i} M_{i}}{\Gamma\left(\sigma_{n}\right)} \max _{t \in J}\left|S_{i(n-1)}-S_{i(m-1)}\right| \int_{0}^{t}(t-\tau)^{\sigma_{n-1}} \mathrm{~d} \tau \\
& \leq \frac{L M T^{\sigma_{n}}}{\sigma_{n} \Gamma\left(\sigma_{n}\right)}\left\|S_{i(n-1)}-S_{i(m-1)}\right\| \leq \alpha \mid S_{i(n-1)}-S_{i(m-1)} \|
\end{aligned}
$$


Let $n=m+1$ then,

$$
\left\|S_{i(m+1)}-S_{i m}\right\| \leq \alpha\left\|S_{i m}-S_{i(m-1)}\right\| \leq \alpha^{2}\left\|S_{i(m-1)}-S_{i(m-2)}\right\| \leq \cdots \leq \alpha^{m}\left\|S_{i 1}-S_{i 0}\right\|
$$

Using the triangle inequality,

$$
\begin{aligned}
\left\|S_{i n}-S_{i m}\right\| & \leq\left\|S_{i(m+1)}-S_{i m}\right\|+\left\|S_{i(m+2)}-S_{i(m+1)}\right\|+\cdots+\left\|S_{i n}-S_{i(n-1)}\right\| \\
& \leq\left[\alpha^{m}+\alpha^{m+1}+\cdots+\alpha^{n-1}\right]\left\|S_{i 1}-S_{i 0}\right\| \\
& \leq \alpha^{m}\left[1+\alpha+\cdots+\alpha^{n-m-1}\right]\left\|S_{i 1}-S_{i 0}\right\| \\
& \leq \alpha^{m}\left[\frac{1-\alpha^{n-m}}{1-\alpha}\right]\left\|y_{i 1}(t)\right\|
\end{aligned}
$$

Since, $0<\alpha<1$, and $n \geq m$ then, $\left(1-\alpha^{n-m}\right) \leq 1$. Consequently,

$$
\left\|S_{i n}-S_{i m}\right\| \leq \frac{\alpha^{m}}{1-\alpha}\left\|y_{i 1}(t)\right\| \leq \frac{\alpha^{m}}{1-\alpha} \max _{t \in J}\left|y_{i 1}(t)\right|
$$

but, $\left|y_{i 1}(t)\right| \leq \infty$ and as $m \rightarrow \infty$ then, $\left\|S_{i n}-S_{i m}\right\| \rightarrow 0$ and hence, $\left\{S_{i n}\right\}$ is a Cauchy sequence in this Banach space so, the series $\sum_{k=0}^{\infty} y_{i k}(t)$ converges and the proof is complete.

\subsection{Error Analysis}

For ADM, we can estimate the maximum absolute truncated error of the Adomian's series solution in the following theorem.

Theorem 3. The maximum absolute truncation error of the series solution (10) to the system (1)-(2) is estimated to be,

$$
\max _{t \in J}\left|y_{i}(t)-\sum_{k=0}^{m} y_{i k}(t)\right| \leq \frac{\alpha^{m}}{1-\alpha} \max _{t \in J}\left|y_{i 1}(t)\right| .
$$

Proof. From Theorem 2 we have,

$$
\left\|S_{i n}-S_{i m}\right\| \leq \frac{\alpha^{m}}{1-\alpha} \max _{t \in J}\left|y_{i 1}(t)\right| .
$$

But, $S_{\text {in }}=\sum_{k=0}^{n} y_{i k}(t)$ as $n \rightarrow \infty$ then, $S_{i n} \rightarrow y_{i}(t)$ so,

$$
\left\|y_{i}(t)-S_{i m}\right\| \leq \frac{\alpha^{m}}{1-\alpha} \max _{t \in J}\left|y_{i 1}(t)\right| .
$$

So, the maximum absolute truncation error in the interval $J$ is,

$$
\max _{t \in J}\left|y_{i}(t)-\sum_{k=0}^{m} y_{i k}(t)\right| \leq \frac{\alpha^{m}}{1-\alpha} \max _{t \in J}\left|y_{i 1}(t)\right|
$$

and this completes the proof.

\section{Numerical Examples}

Example 1. Consider the following nonlinear system of FDEs,

$$
\begin{aligned}
& D^{\alpha} y_{1}=2 y_{2}^{2}, \\
& D^{\alpha} y_{2}=t y_{1}, \\
& D^{\alpha} y_{3}=y_{2} y_{3},
\end{aligned}
$$


subject to the initial conditions,

$$
y_{1}(0)=0, y_{2}(0)=1, y_{3}(0)=1,
$$

where $\alpha \in(0,1)$.

This system was discussed before in [25], it is solved by using the iterative method. Now, we will solve it by using ADM. Applying ADM to system (11) leads to the following recursive relations,

$$
\begin{aligned}
& y_{1,0}=0, \quad y_{1, j+1}=J^{\alpha}\left(2 A_{1, j}\right), \\
& y_{2,0}=1, \quad y_{2, j+1}=J^{\alpha}\left(t y_{1, j}\right), \\
& y_{3,0}=1, \quad y_{3, j+1}=J^{\alpha}\left(A_{2, j}\right),
\end{aligned}
$$

where $A_{1, j}$ and $A_{2, j}$ represent the Adomian polynomials of the nonlinear terms $y_{2}^{2}$ and $y_{2} y_{3}$ respectively.

Using the relations (12)-(14), the first three terms of the series solution when $\alpha=1$ are,

$$
\begin{aligned}
& y_{1}=t+\cdots, \\
& y_{2}=1+\frac{t^{3}}{3}+\cdots, \\
& y_{3}=1+t+\frac{t^{2}}{2}+\cdots .
\end{aligned}
$$

while for $\alpha=0.75$ are,

$$
\begin{aligned}
& y_{1}=\frac{4 t^{3 / 4}}{3 \Gamma(3 / 4)}+\cdots, \\
& y_{2}=1+\frac{32 \Gamma(11 / 4) t^{5 / 2}}{45 \sqrt{\pi} \Gamma(3 / 4)}+\cdots, \\
& y_{3}=1+\frac{4 t^{3 / 4}}{3 \Gamma(3 / 4)}+\frac{4 t^{3 / 2}}{3 \sqrt{\pi}}+\cdots .
\end{aligned}
$$

and for $\alpha=0.5$ are,

$$
\begin{aligned}
& y_{1}=\frac{2 \sqrt{t}}{\sqrt{\pi}}+\cdots, \\
& y_{2}=1+\frac{3 t^{2}}{4}+\cdots, \\
& y_{3}=1+\frac{2 \sqrt{t}}{\sqrt{\pi}}+t+\cdots .
\end{aligned}
$$

Figures 1(a)-(c) show ADM solution of $y_{1}, y_{2}$ and $y_{3}$ at different values of $\alpha \quad(\alpha=1,0.75,0.5,0.25)$.

Example 2. Consider the following nonlinear system of FDEs,

$$
\begin{aligned}
& D^{0.5} y_{1}=\Gamma(1.5)+y_{2}^{2}-t^{4}, \\
& D^{1.5} y_{2}=\frac{\Gamma(3)}{\Gamma(1.5)} y_{1}+y_{1}^{4}-t^{2}, \\
& D^{2.5} y_{3}=\frac{\Gamma(5)}{\Gamma(2.5)} y_{1}^{3},
\end{aligned}
$$



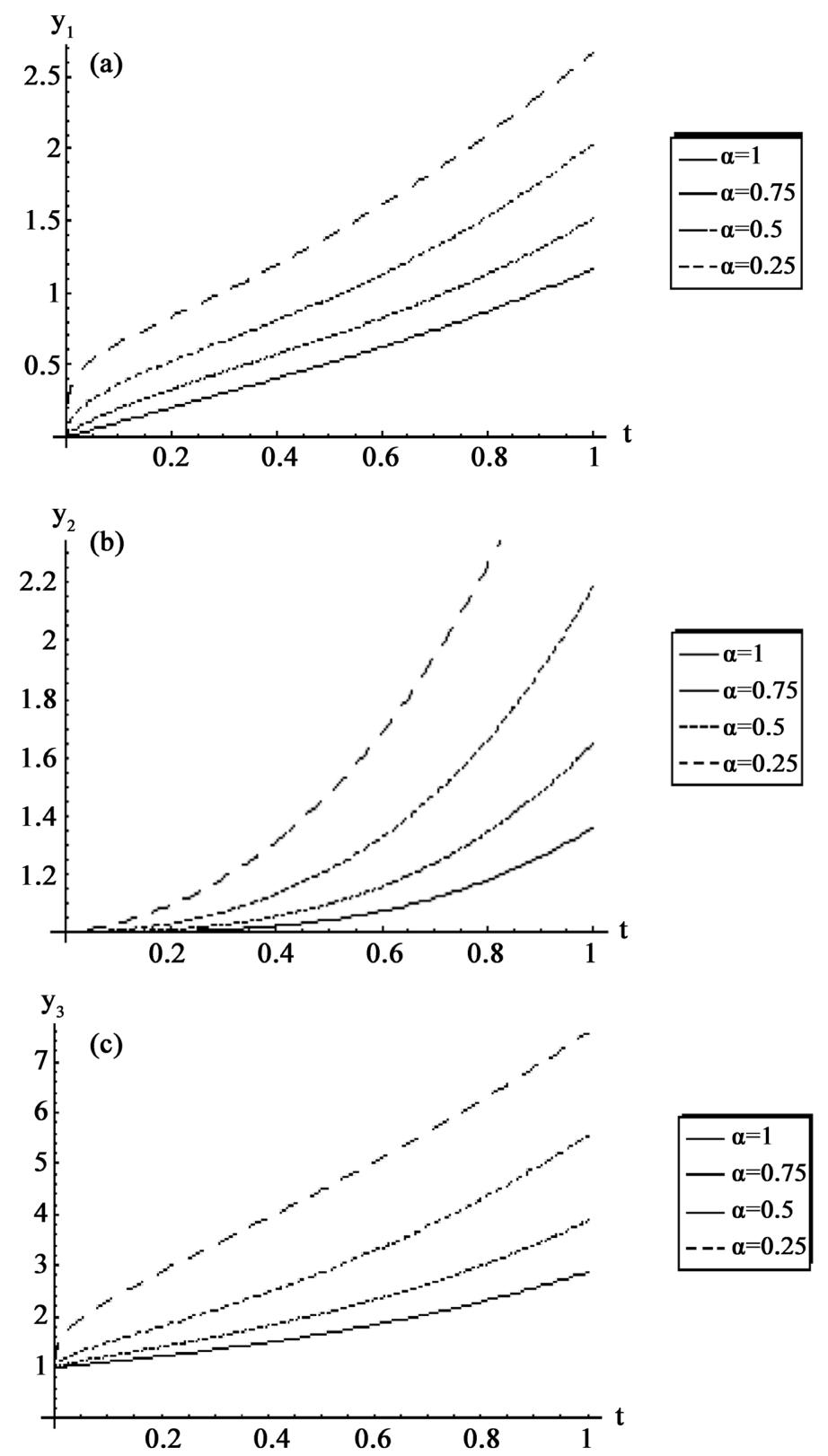

Figure 1. (a) ADM Sol. [ $n=5$ ]; (b) ADM Sol. [ $n=5$ ]; (c) ADM Sol [ $n=5$ ].

subject to the initial conditions,

$$
\begin{aligned}
& y_{1}(0)=0, y_{2}(0)=0, y_{2}^{\prime}(0)=0, \\
& y_{3}(0)=0, y_{3}^{\prime}(0)=0, y_{3}^{\prime \prime}(0)=0,
\end{aligned}
$$

which has the exact solution $y_{1}(t)=t^{0.5}, y_{2}(t)=t^{2}$ and $y_{3}(t)=t^{4}$.

Using ADM to system (18) leads to the following scheme,

$$
\begin{gathered}
y_{1,0}=t^{1 / 2}-\frac{\Gamma(5)}{\Gamma(5.5)} t^{4.5}, \quad y_{1, j+1}=J^{1 / 2}\left(A_{1, j}\right), \\
y_{2,0}=-\frac{\Gamma(3)}{\Gamma(4.5)} t^{3.5}, \quad y_{2, j+1}=\frac{\Gamma(3)}{\Gamma(1.5)} J^{1.5}\left(y_{1, j}\right)+J^{1.5}\left(A_{2, j}\right),
\end{gathered}
$$




$$
y_{3,0}=0, \quad y_{3, j+1}=\frac{\Gamma(5)}{\Gamma(2.5)} J^{2.5}\left(A_{3, j}\right),
$$

where $A_{1, j}, A_{2, j}$ and $A_{3, j}$ represent the Adomian polynomials of the nonlinear terms $y_{2}^{2}, y_{1}^{4}$ and $y_{1}^{3}$ respectively.

Using relations (19)-(21), the first few terms of the series solution are,

$$
\begin{aligned}
y_{1}= & t^{0.5}-0.458516 t^{4.5}+0.0106171 t^{7.5}+\cdots, \\
y_{2}= & -0.171943 t^{3.5}+t^{2}+0.171943 t^{3.5}-0.0752253 t^{6}-0.094092 t^{7.5} \\
& +0.0334503 t^{11.5}-0.00647686 t^{15.5}+0.000523436 t^{19.5}+\cdots, \\
y_{3}= & t^{4}-0.177317 t^{8}+0.0269405 t^{12}-0.00192082 t^{16}+\cdots
\end{aligned}
$$

A comparison between ADM solution and exact solution of $y_{1}, y_{2}$ and $y_{3}$ is given in Figures 2(a)-(c) $(n=10)$.
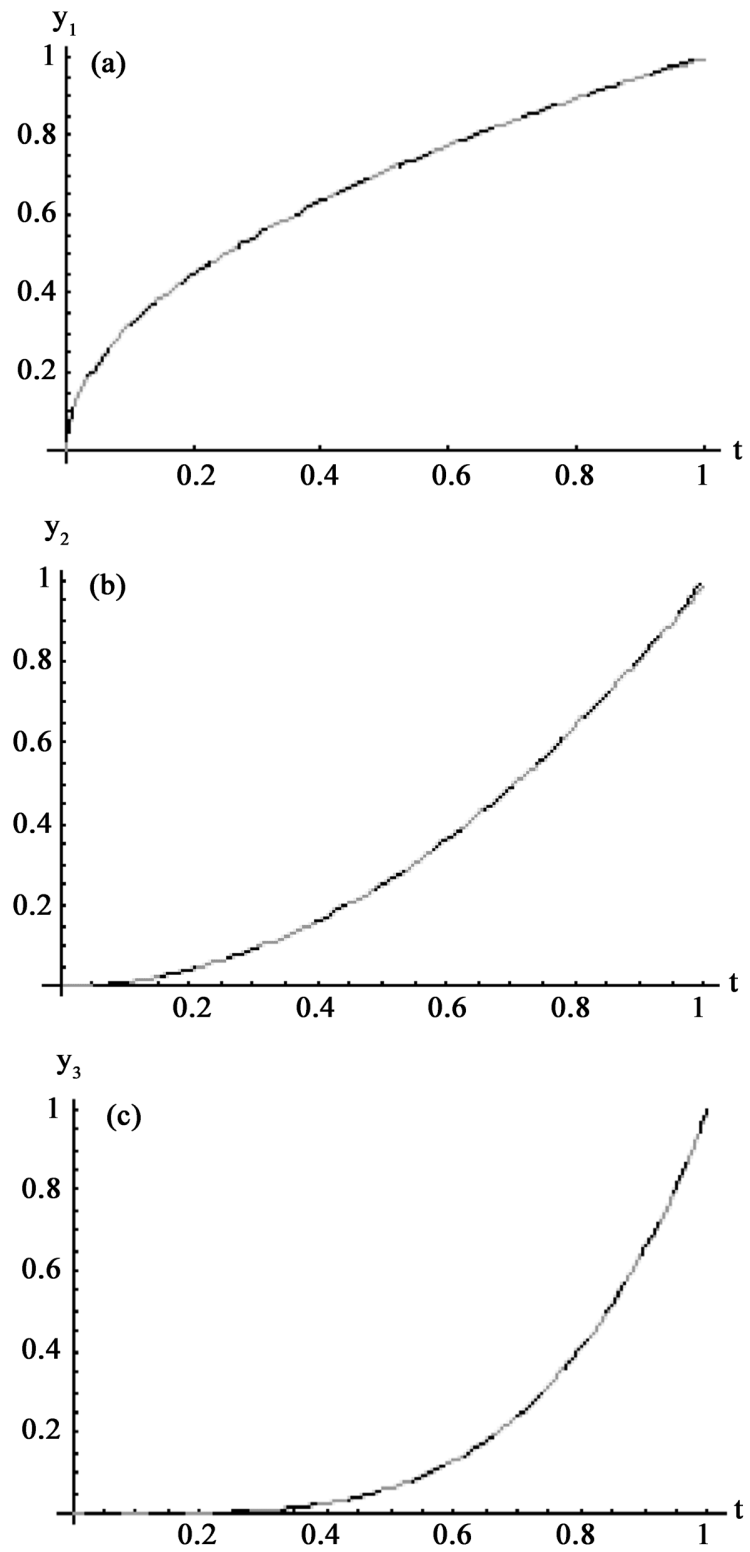

Figure 2. (a) ADM and Exact Sol; (b) ADM and Exact Sol; (c) ADM and Exact Sol. 
Example 3. Consider the following nonlinear system of FDEs [26],

$$
\begin{aligned}
& D^{\alpha} y_{1}=y_{1}^{2}+y_{2}, \\
& D^{\alpha} y_{2}=y_{2} \cos y_{1},
\end{aligned}
$$

subject to the initial conditions,

$$
y_{1}(0)=0, y_{2}(0)=1,
$$

where $\alpha \in(0,1)$.

Using ADM to system (23) leads to the following scheme,

$$
\begin{gathered}
y_{1,0}=0, \quad y_{1, j+1}=J^{\alpha}\left(A_{1, j}\right)+J^{\alpha}\left(y_{2, j}\right), \\
y_{2,0}=1, \quad y_{2, j+1}=J^{\alpha}\left(A_{2, j}\right),
\end{gathered}
$$

where $A_{1, j}$ and $A_{2, j}$ represent the Adomian polynomials of the nonlinear terms $y_{1}^{2}$ and $y_{2} \cos y_{1}$ respectively.

Using the relations (24)-(25), the first four terms of the series solution are,

$$
\begin{gathered}
y_{1}=\frac{t^{\alpha}}{\Gamma(\alpha+1)}+\frac{t^{2 \alpha}}{\Gamma(2 \alpha+1)}+\frac{\Gamma(2 \alpha+1) t^{3 \alpha}}{[\Gamma(\alpha+1)]^{2} \Gamma(3 \alpha+1)}+\cdots, \\
y_{2}=1+\frac{t^{\alpha}}{\Gamma(\alpha+1)}+\frac{t^{2 \alpha}}{\Gamma(2 \alpha+1)}-\frac{\Gamma(2 \alpha+1) t^{3 \alpha}}{[\Gamma(\alpha+1)]^{2} \Gamma(3 \alpha+1)}+\cdots .
\end{gathered}
$$

Figure 3(a) and Figure 3(b) show ADM solution of $y_{1}$ and $y_{2}$ at different
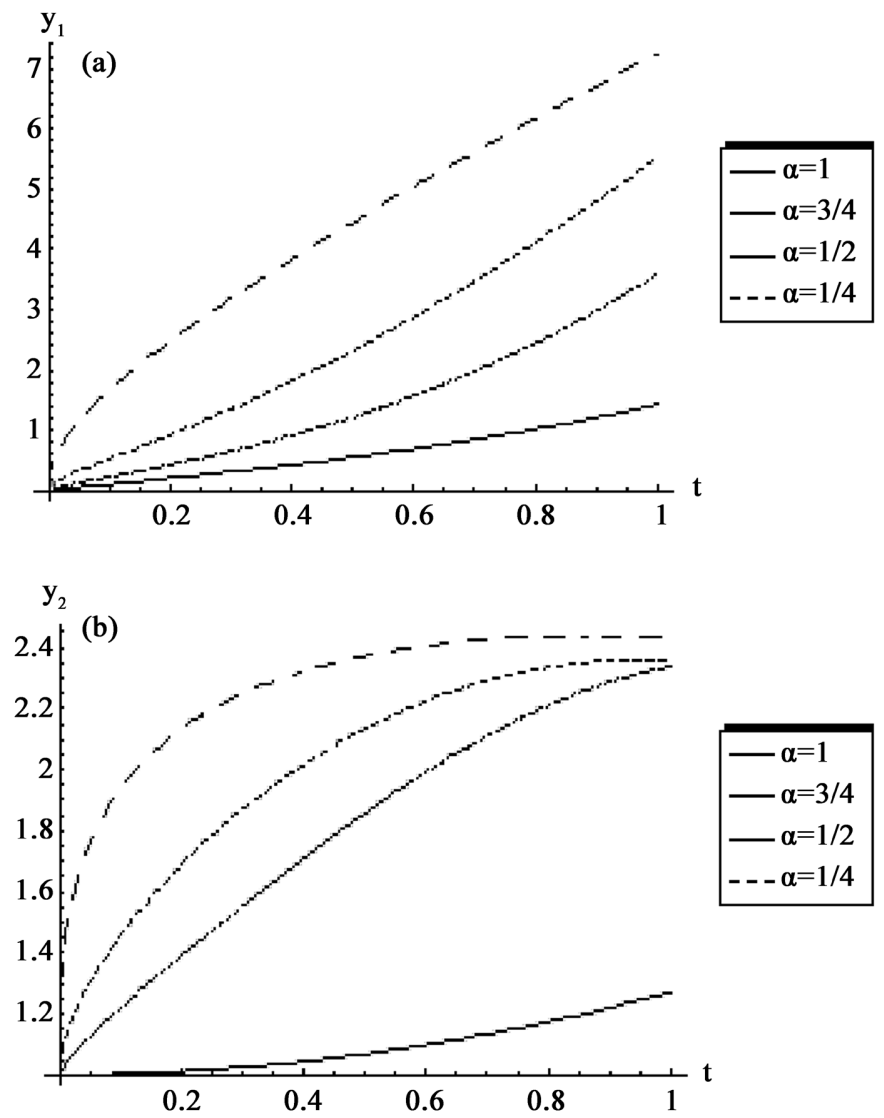

Figure 3. (a) ADM Sol. [ $n=5$ ]; (b) ADM Sol. [ $n=5]$. 
values of $\alpha \quad(\alpha=1,3 / 4,1 / 2,1 / 4)$.

Example 4. Consider the nonlinear system of FDEs,

$$
\begin{aligned}
& D^{3 / 2} y_{1}=\frac{1}{8} y_{2}^{2}+t, \\
& D^{3 / 2} y_{2}=\frac{1}{4} y_{1}^{4}+t^{2}, \quad 0<t \leq 1,
\end{aligned}
$$

subject to the initial conditions,

$$
y_{1}(0)=0, y_{1}^{\prime}(0)=0, y_{2}(0)=0, y_{2}^{\prime}(0)=0 .
$$

Using ADM to the system (28), we get

$$
\begin{aligned}
& y_{1,0}=J^{3 / 2}(t), \quad y_{1, j+1}=\frac{1}{8} J^{3 / 2}\left(A_{1, j}\right), \\
& y_{2,0}=J^{3 / 2}\left(t^{2}\right), \quad y_{2, j+1}=\frac{1}{4} J^{3 / 2}\left(A_{2, j}\right),
\end{aligned}
$$

From the relations (29), the first two terms of the series solution are,

$$
\begin{aligned}
& y_{1}=\left(\frac{8 t^{5 / 2}}{15 \sqrt{\pi}}\right)+\left(\frac{262144 t^{17 / 2}}{1206079875 \sqrt{\pi}}\right)+\cdots, \\
& y_{2}=\left(\frac{32 t^{7 / 2}}{105 \sqrt{\pi}}\right)+\left(\frac{268435456 t^{23 / 2}}{1129407654375 \pi^{3 / 2}}\right)+\cdots .
\end{aligned}
$$

Figure 4(a) and Figure 4(b) show ADM solution of $y_{1}$ and $y_{2} \quad(m=5)$.

Now, we will use Theorem 3 to evaluate the maximum absolute truncated error of the series solution (30). So, we evaluate the following values,

- $L_{1}:\left|f_{1}(y)-f_{1}(z)\right|=\left|y^{2}-z^{2}\right| \leq|y+z||y-z| \leq 2|y-z| \Rightarrow L_{1}=2$.
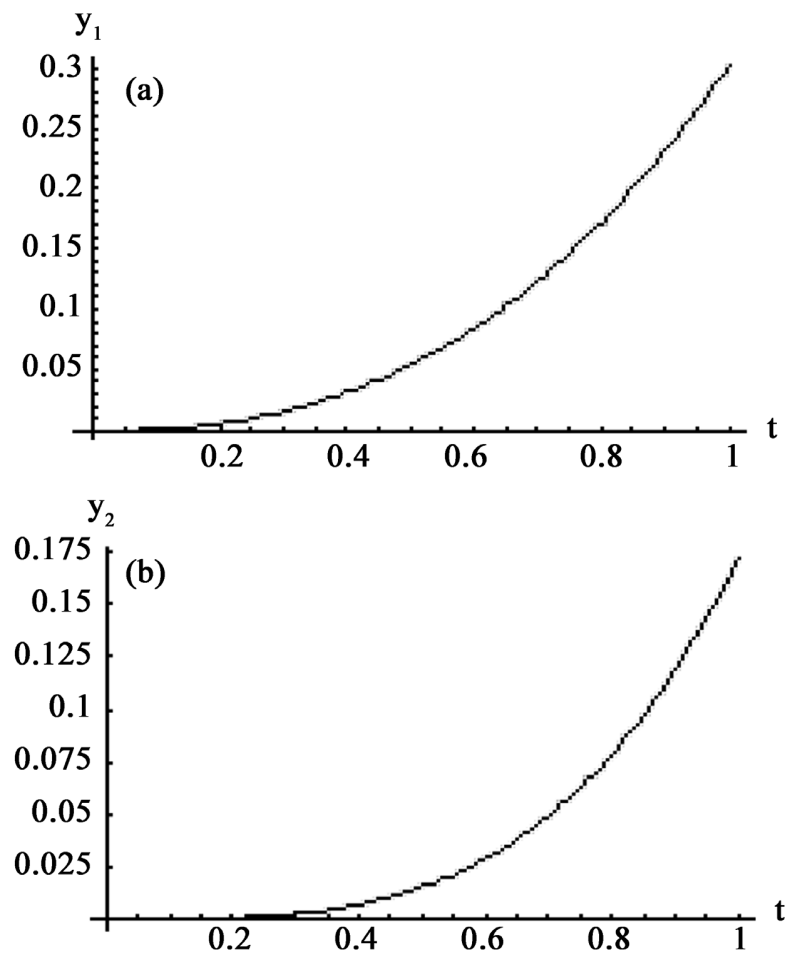

Figure 4. (a) ADM Sol; (b) ADM Sol. 
- $M_{1}:\left|\beta_{1}(\tau)\right| \leq \frac{1}{8} \Rightarrow M_{1}=\frac{1}{8}$.

- $L_{2}:\left|f_{2}(y)-f_{2}(z)\right|=\left|y^{4}-z^{4}\right| \leq\left|y^{2}+z^{2}\right||y+z||y-z| \leq 4|y-z| \Rightarrow L_{2}=4$.

- $M_{2}:\left|\beta_{2}(\tau)\right| \leq \frac{1}{4} \Rightarrow M_{2}=\frac{1}{4}$.

- $\alpha: \alpha=\frac{L M T^{\sigma_{n}}}{\Gamma\left(\sigma_{n}+1\right)}=\frac{1}{\Gamma(5 / 2)}$.

- $\max _{t \in J}\left|y_{11}(t)\right|=\frac{262144}{1206079875 \sqrt{\pi}}, \max _{t \in J}\left|y_{21}(t)\right|=\frac{268435456}{1129407654375 \pi^{3 / 2}}$.

The maximum error of $y_{1}$ :

$\max _{t \in J}\left|y_{1}(t)-\sum_{k=0}^{m} y_{1 k}(t)\right| \leq \frac{\alpha^{m}}{1-\alpha} \max _{t \in J}\left|y_{11}(t)\right|$,

- For $m=5: \max _{t \in J}\left|y_{1}(t)-\sum_{k=0}^{5} y_{1 k}(t)\right| \leq 0.000119234$,

- For $m=10: \max _{t \in J}\left|y_{1}(t)-\sum_{k=0}^{10} y_{1 k}(t)\right| \leq 0.0000287222$,

- For $m=15: \max _{t \in J}\left|y_{1}(t)-\sum_{k=0}^{15} y_{1 k}(t)\right| \leq 6.9189 \times 10^{-6}$,

- For $m=20: \max _{t \in J}\left|y_{1}(t)-\sum_{k=0}^{20} y_{1 k}(t)\right| \leq 1.66669 \times 10^{-6}$.

The maximum error of $y_{2}$ :

$\max _{t \in J}\left|y_{2}(t)-\sum_{k=0}^{m} y_{2 k}(t)\right| \leq \frac{\alpha^{m}}{1-\alpha} \max _{t \in J}\left|y_{21}(t)\right|$,

- For $m=5: \max _{t \in J}\left|y_{2}(t)-\sum_{k=0}^{5} y_{2 k}(t)\right| \leq 0.0000415025$,

- For $m=10: \max _{t \in J}\left|y_{2}(t)-\sum_{k=0}^{10} y_{2 k}(t)\right| \leq 9.99755 \times 10^{-6}$,

- For $m=15: \max _{t \in J}\left|y_{2}(t)-\sum_{k=0}^{15} y_{2 k}(t)\right| \leq 2.40831 \times 10^{-6}$,

- For $m=20: \max _{t \in J}\left|y_{2}(t)-\sum_{k=0}^{20} y_{2 k}(t)\right| \leq 5.80138 \times 10^{-7}$.

\section{Application: On Fractional-Order Rabies Model}

The fractional-order rabies model,

$$
\begin{aligned}
& D^{\alpha} y_{1}=-b y_{1} y_{2}, \\
& D^{\alpha} y_{2}=b y_{1} y_{2}-d y_{2},
\end{aligned}
$$

subject to the initial conditions,

$$
y_{1}(0)=1, y_{2}(0)=2,
$$

was discussed before in [27], it was solved by using Adams-type predictor-corrector method. Now, we will solve it by using ADM.

Applying ADM to the system (31) leads to the following scheme,

$$
\begin{gathered}
y_{1,0}=1, \quad y_{1, j+1}=-b J^{\alpha}\left[A_{1, j}\right], \\
y_{2,0}=2, \quad y_{2, j+1}=J^{\alpha}\left[A_{1, j}-3 y_{2, j}\right],
\end{gathered}
$$

where $A_{1, j}$ represents the Adomian polynomials of the nonlinear term $y_{1} y_{2}$. 
Using the relations (32)-(33) and taking $b=1, d=3, \alpha=0.9$, the first five-terms of the series solution are,
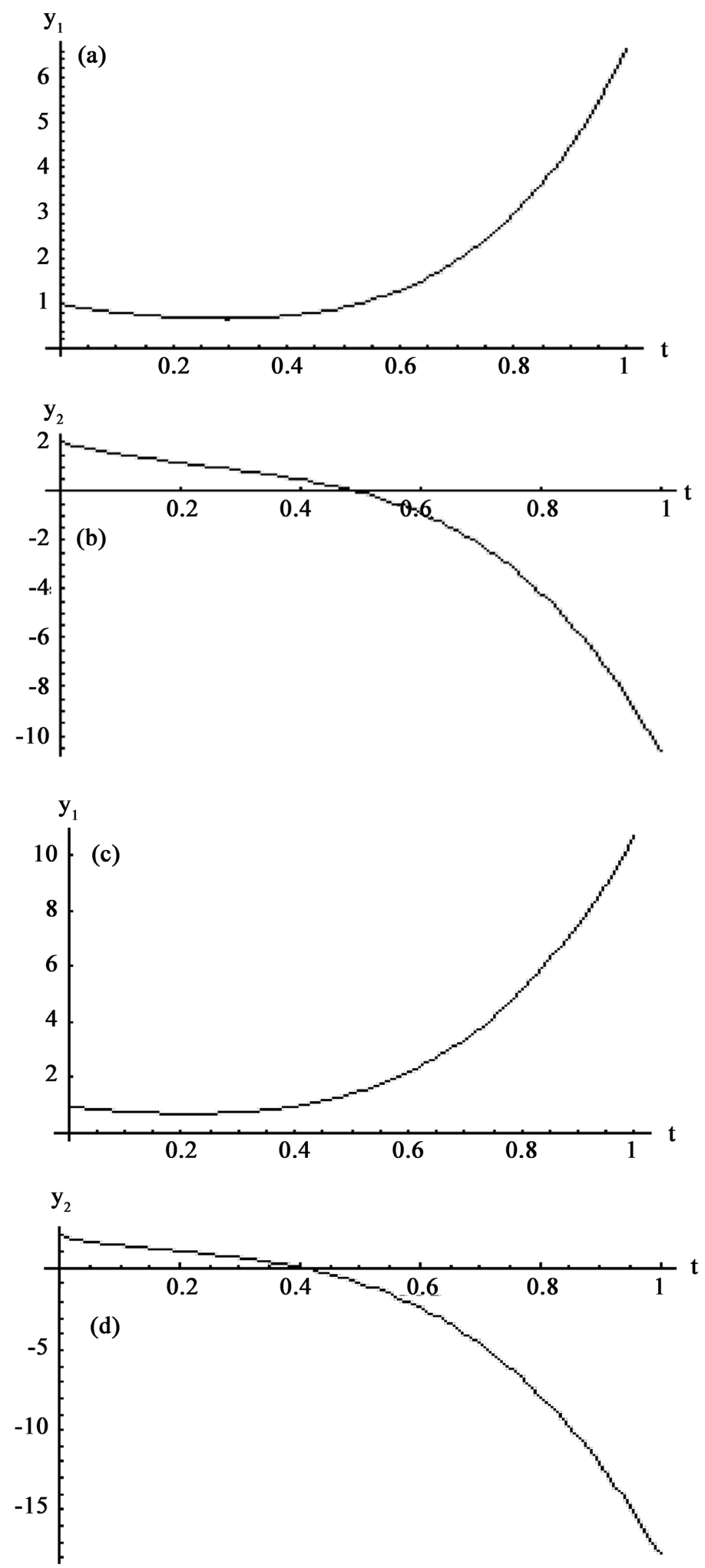

Figure 5. (a) ADM Sol. [ $\alpha=0.9$ ]; (b) ADM Sol. [ $\alpha=0.9$ ]; (c) ADM Sol. [ $\alpha=0.8$ ]; (d) ADM Sol. [ $\alpha=0.8$. 


$$
\begin{aligned}
& y_{1}=1-2.07951 t^{0.9}+4.77187 t^{1.8}-8.27196 t^{2.7}+11.207 t^{3.6}+\cdots, \\
& y_{2}=2-4.15902 t^{0.9}+2.38594 t^{1.8}+5.39472 t^{2.7}-16.2513 t^{3.6}+\cdots .
\end{aligned}
$$

while for $\alpha=0.8$, the series solution will be,

$$
\begin{aligned}
& y_{1}=1-2.14734 t^{0.8}+5.59587 t^{1.6}-11.1311 t^{2.4}+17.371 t^{3.2}+\cdots, \\
& y_{2}=2-4.29469 t^{0.8}+2.79794 t^{1.6}+7.10592 t^{2.4}-25.5643 t^{3.2}+\cdots .
\end{aligned}
$$

Figures 5(a)-(d) show ADM solution of $y_{1}$ and $y_{2} \quad(n=5)$ at different values of $\alpha$.

\section{Conclusion}

In this paper, we use a simple method to solve nonlinear system of FDEs, this method gives a good approximate analytical solution of this type of equation as we compare ADM solution with the exact solution and also by evaluating the maximum absolute error which results from using partial sum of the series ADM solution.

\section{Conflicts of Interest}

The author declares no conflicts of interest regarding the publication of this paper.

\section{References}

[1] Miller, K.S. and Ross, B. (1993) An Introduction to the Fractional Calculus and Fractional Differential Equations. Wiley-Interscience, New York.

[2] Podlubny, I. (1999) Fractional Differential Equations. Academic Press, New York.

[3] Kilbas, A.A., Srivastava, H.M. and Trujillo, J.J. (2006) Theory and Applications of Fractional Differential Equations. Elsevier, New York.

[4] Abd El-Salam, Sh.A. and El-Sayed, A.M.A. (2007) On the stability of some fractional-order non-autonomous systems. Electronic Journal of Qualitative Theory of Differential Equations, No. 6, 1-14. https://doi.org/10.14232/ejqtde.2007.1.6

[5] El-Sayed, A.M.A. and Abd El-Salam, Sh.A. (2008) On the Stability of a Fractional-Order Differential Equation with Nonlocal Initial Condition. Electronic Journal of Qualitative Theory of Differential Equations, No. 29, 1-8. https://doi.org/10.14232/ejqtde.2008.1.29

[6] Evans, D.J. and Raslan, K.R. (2005) The Adomian Decomposition Method for Solving Delay Differential Equation. International Journal of Computer Mathematics, 82, 49-54. https://doi.org/10.1080/00207160412331286815

[7] Zwillinger, D. (1997) Handbook of Differential Equations. Academic Press, USA.

[8] Mensour, B. and Longtin, A. (1998) Chaos Control in Multistable Delay-Differential Equations and Their Singular Limit Maps. Physical Review E, 58, 410-422. https://doi.org/10.1103/PhysRevE.58.410

[9] Hefferan, J.M. and Corless, R.M. (2005) Solving Some Delay Differential Equations with Computer Algebra. Applied Probability Trust, No. 1, 1-22.

[10] El-Sayed, A.M.A., El-Mesiry, E.M. and El-Saka, H.A.A. (2004) Numerical Solution for Multi-Term Fractional (Arbitrary) Orders Differential Equations. Computational and Applied Mathematics, 23, 33-54.

[11] El-Mesiry, E.M., El-Sayed, A.M.A. and El-Saka, H.A.A. (2005) Numerical Methods 
for Multi-Term Fractional (Arbitrary) Orders Differential Equations. Applied Mathematics and Computation, 160, 683-699. https://doi.org/10.1016/j.amc.2003.11.026

[12] Alam Khan, N., Abdul Razzaq, O., Ara, A. and Riaz, F. (2016) Numerical Solution of System of Fractional Differential Equations in Imprecise Environment. In: López-Ruiz, R., Ed., Numerical Simulation: From Brain Imaging to Turbulent Flows, IntechOpen, London, 167-186. https://doi.org/10.5772/64150

[13] Atangana, A. and Alabaraoye, E. (2013) Solving a system of Fractional Partial Differential Equations Arising in the Model of HIV Infection of $\mathrm{CD}^{+}$Cells and Attractor One-Dimensional Keller-Segel Equations. Advances in Difference Equations, 94, Article No. 94. https://doi.org/10.1186/1687-1847-2013-94

[14] Hammad, H.A. and De la Sen, M. (2021) Tripled Fixed Point Techniques for Solving System of Tripled-Fractional Differential Equations. AIMS Mathematics, 6, 2330-2343. https://doi.org/10.3934/math.2021141

[15] Rida, S.Z. and Arafa, A.A.M. (2011) New Method for Solving Linear Fractional Differential Equations. International Journal of Differential Equations, 2011, Article ID: 814132. https://doi.org/10.1155/2011/814132

[16] Daraghmeh, A., Qatanani, N. and Saadeh, A. (2020) Numerical Solution of Fractional Differential Equations. Applied Mathematics, 11, 1100-1115.

https://doi.org/10.4236/am.2020.1111074

[17] Adomian, G. (1994) Solving Frontier Problems of Physics: The Decomposition Method. Springer, Dordrecht. https://doi.org/10.1007/978-94-015-8289-6

[18] Adomian, G. (1983) Stochastic System. Academic Press, Cambridge.

[19] Adomian, G. (1986) Nonlinear Stochastic Operator Equations. Academic Press, San Diego. https://doi.org/10.1016/C2013-0-10271-1

[20] Adomian, G. (1989) Nonlinear Stochastic Systems: Theory and Applications to Physics. Springer, Dordrecht.

[21] Abbaoui, K. and Cherruault, Y. (1994) Convergence of Adomian's Method Applied to Differential Equations. Computers \& Mathematics with Applications, 28, 103-109. https://doi.org/10.1016/0898-1221(94)00144-8

[22] Cherruault, Y., Adomian, G., Abbaoui, K. and Rach, R. (1995) Further Remarks on Convergence of Decomposition Method. International Journal of Bio-Medical Computing, 38, 89-93. https://doi.org/10.1016/0020-7101(94)01042-Y

[23] Shawaghfeh, N.T. (2002) Analytical Approximate Solution for Nonlinear Fractional Differential Equations. Applied Mathematics and Computation, 131, 517-529. https://doi.org/10.1016/S0096-3003(01)00167-9

[24] El-Kalla, I.L. (2008) Convergence of the Adomian Method Applied to a Class of Nonlinear Integral Equations. Applied Mathematics Letters, 21, 372-376. https://doi.org/10.1016/j.aml.2007.05.008

[25] Momani, S. and Al-Khaled, K. (2005) Numerical Solutions for Systems of Fractional Differential Equations by the Decomposition Method. Applied Mathematics and Computation, 162, 1351-1365. https://doi.org/10.1016/j.amc.2004.03.014

[26] Jafari, H. and Daftardar-Gejji, V. (2006) Solving a System of Nonlinear Fractional Differential Equations Using Adomian Decomposition Method. Journal of Computational and Applied Mathematics, 196, 644-651. https://doi.org/10.1016/j.cam.2005.10.017

[27] Ahmed, E., El-Sayed, A.M.A. and El-Saka, H.A.A. (2007) Equilibrium Points, Stability and Numerical Solutions of Fractional-Order Predator-Prey and Rabies Models. Journal of Mathematical Analysis and Applications, 325, 542-553.

https://doi.org/10.1016/j.jmaa.2006.01.087 